
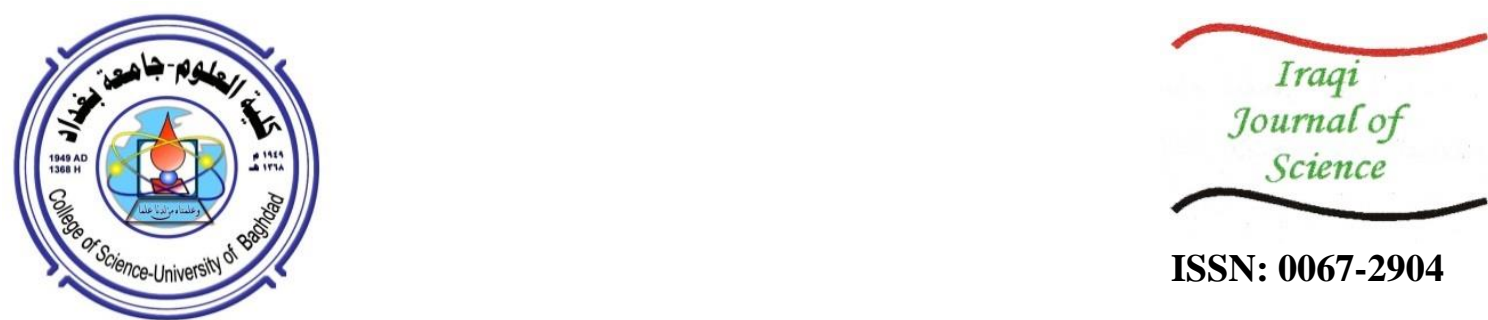

ISSN: 0067-2904

\title{
Recursive Prediction for Lossless Image Compression
}

\author{
Rana Talib Al-Timimi \\ Department of Finance and Banking Science, College of Economics and administration, Mustansiriyah \\ University, Baghdad, Iraq
}

Received: 6/7/2019

Accepted: 4/1/2021

\begin{abstract}
This paper introduced an algorithm for lossless image compression to compress natural and medical images. It is based on utilizing various casual fixed predictors of one or two dimension to get rid of the correlation or spatial redundancy embedded between image pixel values then a recursive polynomial model of a linear base is used.

The experimental results of the proposed compression method are promising in terms of preserving the details and the quality of the reconstructed images as well improving the compression ratio as compared with the extracted results of a traditional linear predicting coding system.
\end{abstract}

Keywords: Polynomial Coding, Lossless Image Compression, Fixed Predictor, Image Compression.
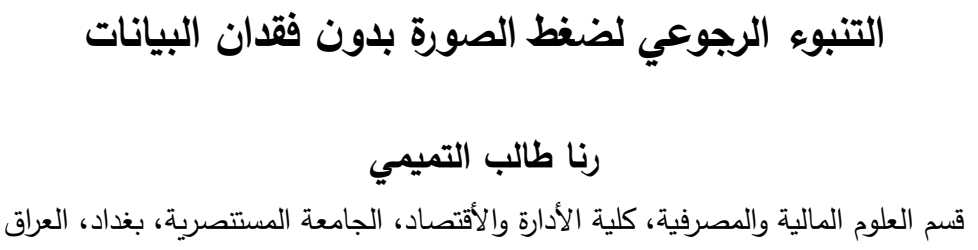

الخلاصة

قدم هذا البحث خوارزمية لضغط الصور بدون فقدان البيانات لضغط الصور الطبيعية والطبية. وهو يعتمد

على استخدام العديد من المتنبئات الثابتة العرضية ذات البعد أو البعدين للتخلص من الارتباط أو التكرار المكاني المضمن بين قيم بكسل الصورة ثم تم استخدام النموذج الخطي متعدد الحدود.

النتائج التجريبية لطريقة الضغط المقترحة واعدة من حيث الحفاظ على التفاصيل وجودة الصور المعاد بناؤها وكذلك تحسين نسبة الضغط مقارنة بالنتائج المستخرجة من نظام الترميز التنبئي الخطي التقليدي.

\section{Introduction}

Image compression techniques aims to eliminate the redundancy in an image in order to store or transmit over the network in an efficient way. These techniques can be categorized into lossless and lossy. In Lossy compression there is a high loss of image information while in lossless the reconstructed image will be exactly the same as the original but it achieves low compression ratio; this consequence of the fact of exploiting the statistical redundancy only (i.e. exploits the coding redundancy and/or inter pixel redundancy) [1-2].

Over the last years, so many researchers in the field of image compression were competing to design efficient algorithms to improve the compression ratio as well the quality of the

\footnotetext{
*Email: rana_talib@uomustansiriyah.edu.iq
} 
reconstructed images by merging several coding techniques. Review on image compressions techniques can be found in [3-5].

The first order Taylor series linear polynomial coding based on modelling the distance between image pixels and the centre was used effectively to compress images by several researchers to remove the redundancy between image pixel neighbours; further information can be found in [6-10].

The primary objective of this paper is to design an efficient algorithm to compress lossless images by combining the fixed predictor with a recursive polynomial model of a linear base.

Materials and Methods

All the estimated predictors in this study as illustrated in table (1) were casual; i.e. pixel (i,j) will be predicted from the previous visited pixels as shown in Figure 1 [11,12]; on the other hand; a recursive polynomial coding or top-down levels scheme is adopted to remove the redundancy embedded within the coefficients to improve the compression ratio and preserve image quality, it worked reversely from subsequent levels to construct up layers [2,13]. Figure 2 illustrates the layout of the suggested compression algorithm.

Table 1-The Models of the tested Predictors

\begin{tabular}{|c|c|c|}
\hline Index & Predictor & Description \\
\hline 1 & $\mathrm{x}(\mathrm{i}-1, \mathrm{j}-1)$ & First order, one dimension Predictor \\
\hline 2 & $\mathrm{x}(\mathrm{i}-1, \mathrm{j})$ & First order, one dimension Predictor \\
\hline 3 & $(\mathrm{x}(\mathrm{i}, \mathrm{j}-1)+\mathrm{x}(\mathrm{i}-1, \mathrm{j})) / 2$ & second order, two dimension Predictor \\
\hline 4 & $\mathrm{x}(\mathrm{i}, \mathrm{j}-1)+(\mathrm{x}(\mathrm{i}-1, \mathrm{j})-\mathrm{x}(\mathrm{i}-1, \mathrm{j}-1)) / 2$ & Third order, two dimension Predictor \\
\hline 5 & $\mathrm{x}(\mathrm{i}-1, \mathrm{j})+(\mathrm{x}(\mathrm{i}, \mathrm{j}-1)-\mathrm{x}(\mathrm{i}-1, \mathrm{j}-1)) / 2$ & Third order, two dimension Predictor \\
\hline 6 & $\mathrm{x}(\mathrm{i}, \mathrm{j}-1)+\mathrm{x}(\mathrm{i}-1, \mathrm{j})-\mathrm{x}(\mathrm{i}-1, \mathrm{j}-1)$ & Third order, two dimension Predictor \\
\hline 7 & $(\mathrm{x}(\mathrm{i}, \mathrm{j}-1)+\mathrm{x}(\mathrm{i}-1, \mathrm{j})+\mathrm{x}(\mathrm{i}-1, \mathrm{j}-1)+\mathrm{x}(\mathrm{i}-1, \mathrm{j}+1)) / 4$ & Fourth order, two dimension Predictor \\
\hline
\end{tabular}

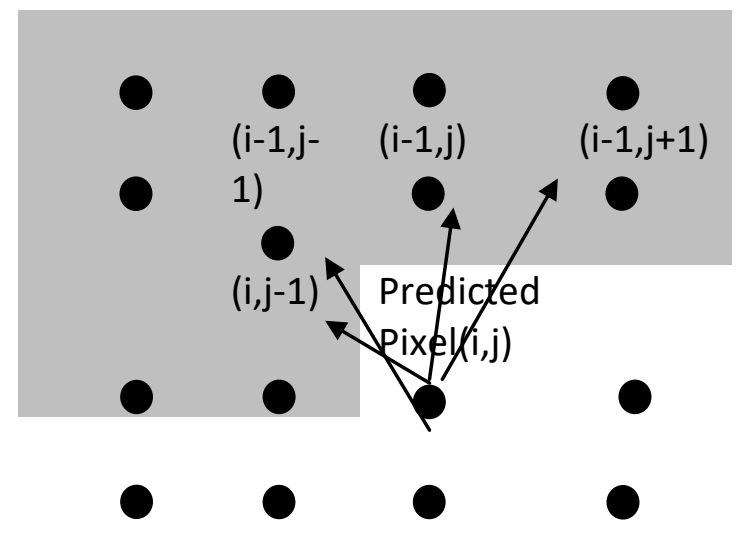

Figure 1 - The Predicted Pixel and the Casual Region [11] 


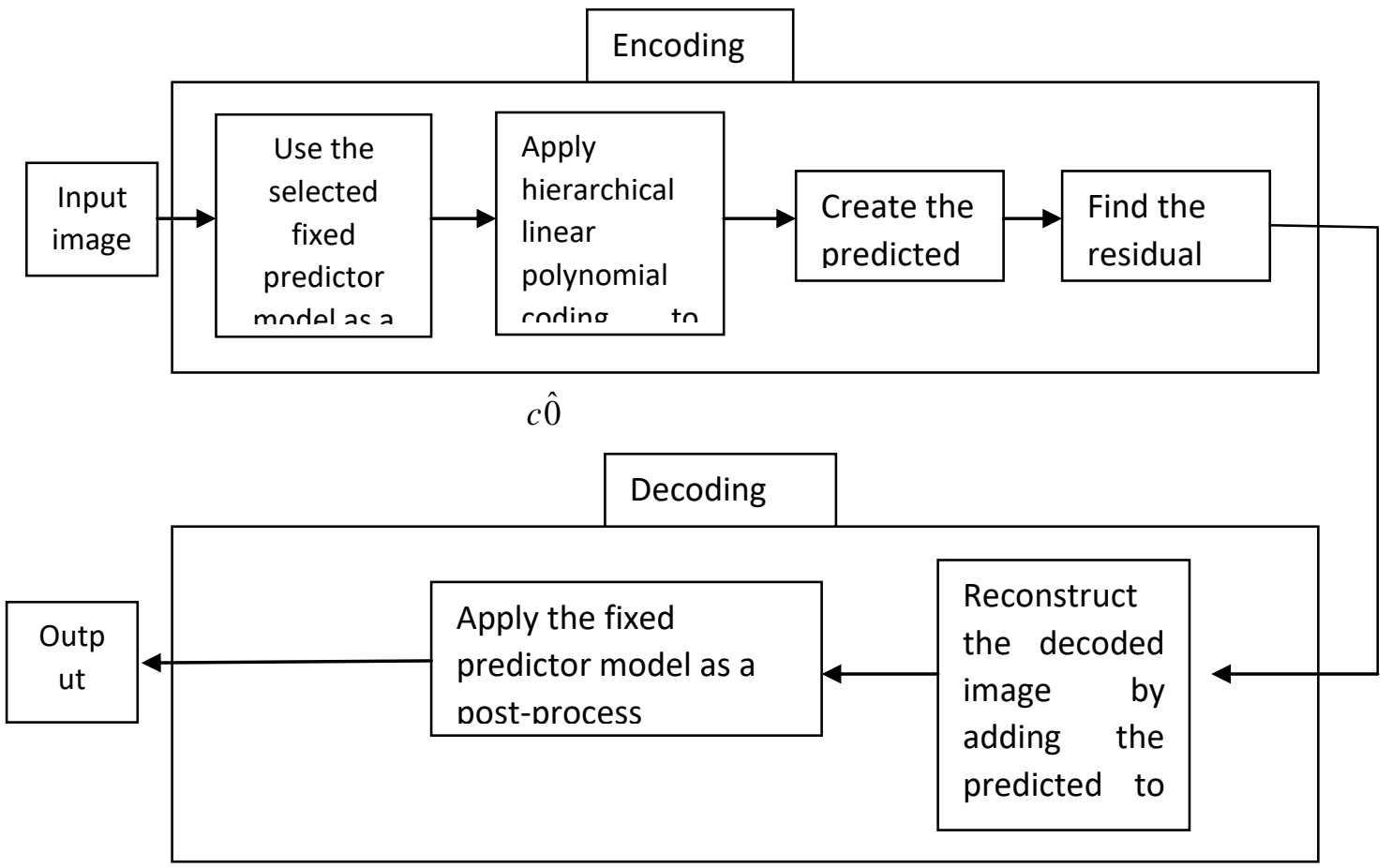

Figure 2- The suggested Compression System

The proposed algorithm was implemented on each predictor model in table (1) separately as follows:

Step 1- Apply the predictor model on the input image; name the output image Ip.

Step 2- A recursive polynomial linear model is applied on image Ip such that two layers will be generated.

In layer 1 ; three coefficients are calculated namely $c_{0}, c_{1}$ and $c_{2}$ using equations 1,2 and 3 $[5,6]$.

$$
\begin{gathered}
c_{0}=\frac{\sum_{i} \sum_{j} \operatorname{Ip}(i, j)}{n \times n} \\
c_{1}=\frac{\sum_{i} \sum_{j} I p(i, j) *(j-x x)}{\sum_{i} \sum_{j}(j-x x)^{2}} \\
c_{2}=\frac{\sum_{i} \sum_{j} I p(i, j) *(i-y y)}{\sum_{i} \sum_{j}(i-y y)^{2}}
\end{gathered}
$$

Where

$\mathrm{n}$ is the image block size

$$
\begin{aligned}
& x x=y y=\frac{n-1}{2} \\
& \mathrm{i}=\mathrm{j}=0, \ldots . \mathrm{n}-1
\end{aligned}
$$

In layer2 another three coefficients will be generated c00, $\mathrm{c} 01$ and $\mathrm{c} 02$ depending on the $\mathrm{c} 0$ coefficient in layer 1 using equations 4,5 and 6 [13].

$$
c 00=\frac{1}{n \times n} \sum_{i} \sum_{j} c 0(i, j)
$$




$$
\begin{gathered}
c 01=\frac{\sum_{i} \sum_{j} c 0(i, j) \times(j-x x)}{\sum_{i} \sum_{j}(j-x x)^{2}} \\
c 02=\frac{\sum_{i} \sum_{j} c 0(i, j) \times(i-y y)}{\sum_{i} \sum_{j}(i-y y)^{2}}
\end{gathered}
$$

For Layer 2:

a- Determine the deterministic part (function formula) $c \tilde{0}[13]$.

$$
c \tilde{0}=c 00+c 01(j-x x)+c 02(i-y y)
$$

b-Find residual [13].

$$
c 0 \operatorname{Re} s d=c 0-c \tilde{0}
$$

c- Build the modeled approximated $c \hat{0}[13]$.

$$
c \hat{0}=c \tilde{0}-c 0 \operatorname{Re} s d
$$

Step 3- Determine the deterministic part $L \widetilde{L}$ [14].

Step 4-Find the error (residual) [14].

$$
L \tilde{L}=c \hat{0}+c 1(j-x x)+c 2(i-y y)
$$

$$
\text { LL Re } s d=\text { Ip }- \text { L } \tilde{L}
$$

Reconstruct the compressed image using the following steps:

a - find the approximation model using [14].:

$$
L L a l l=L \tilde{L}+L L \text { Re } s d
$$

b-Build the decoded image $[10,12]$ :

$$
L \hat{L}(i, j)=\operatorname{LLall}(i, j)+\operatorname{Ip}(i-1, j)
$$

Where $i=2 \ldots N / 2 \quad j=1 \ldots N / 2$

\section{Results}

In this work, all the tested natural and medical images (as indicated in Figure 3 ) were of size 65536 bytes. The tests were done using block size of $8 * 8$. To measure the efficiency of the lossless compression method; the compression ratio was determined (which is the ratio of the original image size to the compressed size). Table 2 illustrates the results of a traditional coding system and a recursive polynomial linear base without applying the predictors; while Table 3 summarizes the compressed image size and the compression ratio obtained by applying the predictors models listed in table 1 and the recursive linear model.

Any way, it obvious that the suggested recursive linear model (with or without using the predictor) is better than the traditional coding system where the compression ratio was improved.

Figure 4 records the resulting images of the suggested recursive prediction using the predictors in Table 1. 


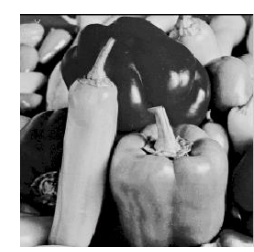

a

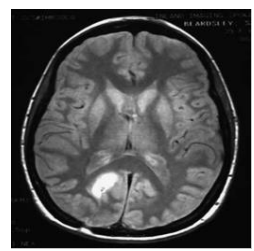

C

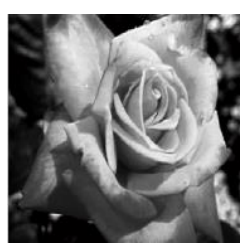

b

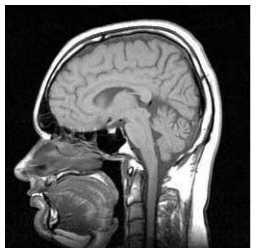

d

Figure 3- Input Images : (a) Pepper (b) Rose (c) Brain1 and (d) Brain2

Table 2 - Compression Performance of a Traditional Linear System and Recursive Prediction

\begin{tabular}{|c|c|c|c|c|}
\hline & \multicolumn{2}{|c|}{$\begin{array}{c}\text { Performance of a Traditional Linear } \\
\text { System }\end{array}$} & \multicolumn{2}{|c|}{$\begin{array}{c}\text { Performance of the Recursive Linear Polynomial } \\
\text { without using any predictor }\end{array}$} \\
\hline Test image & Size & Compression Ratio & Size & Compression Ratio \\
\hline Pepper & 28674 & 2.2856 & 7391 & 8.8670 \\
\hline Rose & 26622 & 2.4617 & 6116 & 10.7155 \\
\hline Brain1 & 6967 & 2.2207 & 4150 & 9.4066 \\
\hline Brain2 & 28290 & 2.3166 & 8443 & 7.7622 \\
\hline
\end{tabular}

Table 3 - Performance of the Recursive Prediction using the predictor

\begin{tabular}{|c|c|c|c|c|c|c|c|c|c|c|c|c|c|c|}
\hline & & dictor1 & & dictor2 & & dictor3 & & dictor4 & & dictor5 & & dictor6 & & dictor7 \\
\hline $\begin{array}{l}\text { Im } \\
\text { age }\end{array}$ & $\begin{array}{l}\mathrm{Si} \\
\mathrm{ze}\end{array}$ & $\begin{array}{c}\text { Comp } \\
\text { ression } \\
\text { Ratio }\end{array}$ & $\begin{array}{l}\mathrm{Si} \\
\mathrm{ze}\end{array}$ & $\begin{array}{c}\text { Comp } \\
\text { ression } \\
\text { Ratio }\end{array}$ & $\begin{array}{l}\mathrm{Si} \\
\mathrm{ze}\end{array}$ & $\begin{array}{c}\text { Comp } \\
\text { ression } \\
\text { Ratio }\end{array}$ & $\begin{array}{l}\text { Si } \\
\text { ze }\end{array}$ & $\begin{array}{c}\text { Comp } \\
\text { ression } \\
\text { Ratio }\end{array}$ & $\begin{array}{l}\mathrm{Si} \\
\mathrm{ze}\end{array}$ & $\begin{array}{c}\text { Comp } \\
\text { ression } \\
\text { Ratio }\end{array}$ & $\begin{array}{l}\text { Si } \\
\text { ze }\end{array}$ & $\begin{array}{c}\text { Comp } \\
\text { ression } \\
\text { Ratio }\end{array}$ & $\begin{array}{l}\text { Si } \\
\text { ze }\end{array}$ & $\begin{array}{c}\text { Comp } \\
\text { ression } \\
\text { Ratio }\end{array}$ \\
\hline $\begin{array}{l}\text { Pep } \\
\text { per }\end{array}$ & $\begin{array}{l}65 \\
24\end{array}$ & $\begin{array}{c}10.045 \\
4\end{array}$ & $\begin{array}{l}64 \\
42\end{array}$ & $\begin{array}{c}10.173 \\
2\end{array}$ & $\begin{array}{l}56 \\
24\end{array}$ & $\begin{array}{c}11.652 \\
9\end{array}$ & $\begin{array}{l}62 \\
32\end{array}$ & $\begin{array}{c}10.516 \\
0\end{array}$ & $\begin{array}{l}64 \\
66\end{array}$ & $\begin{array}{c}10.135 \\
5\end{array}$ & $\begin{array}{l}70 \\
88\end{array}$ & 9.2460 & $\begin{array}{l}55 \\
28\end{array}$ & $\begin{array}{c}11.855 \\
3\end{array}$ \\
\hline $\begin{array}{l}\text { Ro } \\
\text { se }\end{array}$ & $\begin{array}{l}52 \\
02\end{array}$ & $\begin{array}{c}12.598 \\
2\end{array}$ & $\begin{array}{l}51 \\
88\end{array}$ & $\begin{array}{c}12.632 \\
2\end{array}$ & $\begin{array}{l}46 \\
10\end{array}$ & $\begin{array}{c}14.216 \\
1\end{array}$ & $\begin{array}{l}50 \\
64\end{array}$ & $\begin{array}{c}12.941 \\
5\end{array}$ & $\begin{array}{l}50 \\
60\end{array}$ & $\begin{array}{c}12.951 \\
8\end{array}$ & $\begin{array}{l}54 \\
32\end{array}$ & $\begin{array}{c}12.064 \\
8\end{array}$ & $\begin{array}{l}44 \\
22\end{array}$ & $\begin{array}{c}14.820 \\
4\end{array}$ \\
\hline $\begin{array}{l}\text { Bra } \\
\text { in1 }\end{array}$ & $\begin{array}{l}61 \\
36\end{array}$ & $\begin{array}{c}10.680 \\
6\end{array}$ & $\begin{array}{l}60 \\
44\end{array}$ & $\begin{array}{c}10.843 \\
2\end{array}$ & $\begin{array}{l}55 \\
98\end{array}$ & $\begin{array}{c}11.707 \\
0\end{array}$ & $\begin{array}{l}59 \\
02\end{array}$ & $\begin{array}{c}11.104 \\
0\end{array}$ & $\begin{array}{l}58 \\
70\end{array}$ & $\begin{array}{c}11.164 \\
6\end{array}$ & $\begin{array}{l}61 \\
88\end{array}$ & $\begin{array}{c}10.590 \\
8\end{array}$ & $\begin{array}{l}53 \\
50\end{array}$ & $\begin{array}{c}12.249 \\
7\end{array}$ \\
\hline $\begin{array}{l}\text { Bra } \\
\text { in2 }\end{array}$ & $\begin{array}{l}77 \\
26\end{array}$ & 8.4825 & $\begin{array}{l}76 \\
68\end{array}$ & 8.5467 & $\begin{array}{l}66 \\
66\end{array}$ & 9.8314 & $\begin{array}{l}72 \\
42\end{array}$ & 9.0494 & $\begin{array}{l}74 \\
74\end{array}$ & 8.7685 & $\begin{array}{l}82 \\
60\end{array}$ & 7.9341 & $\begin{array}{l}61 \\
96\end{array}$ & $\begin{array}{c}10.577 \\
1\end{array}$ \\
\hline
\end{tabular}




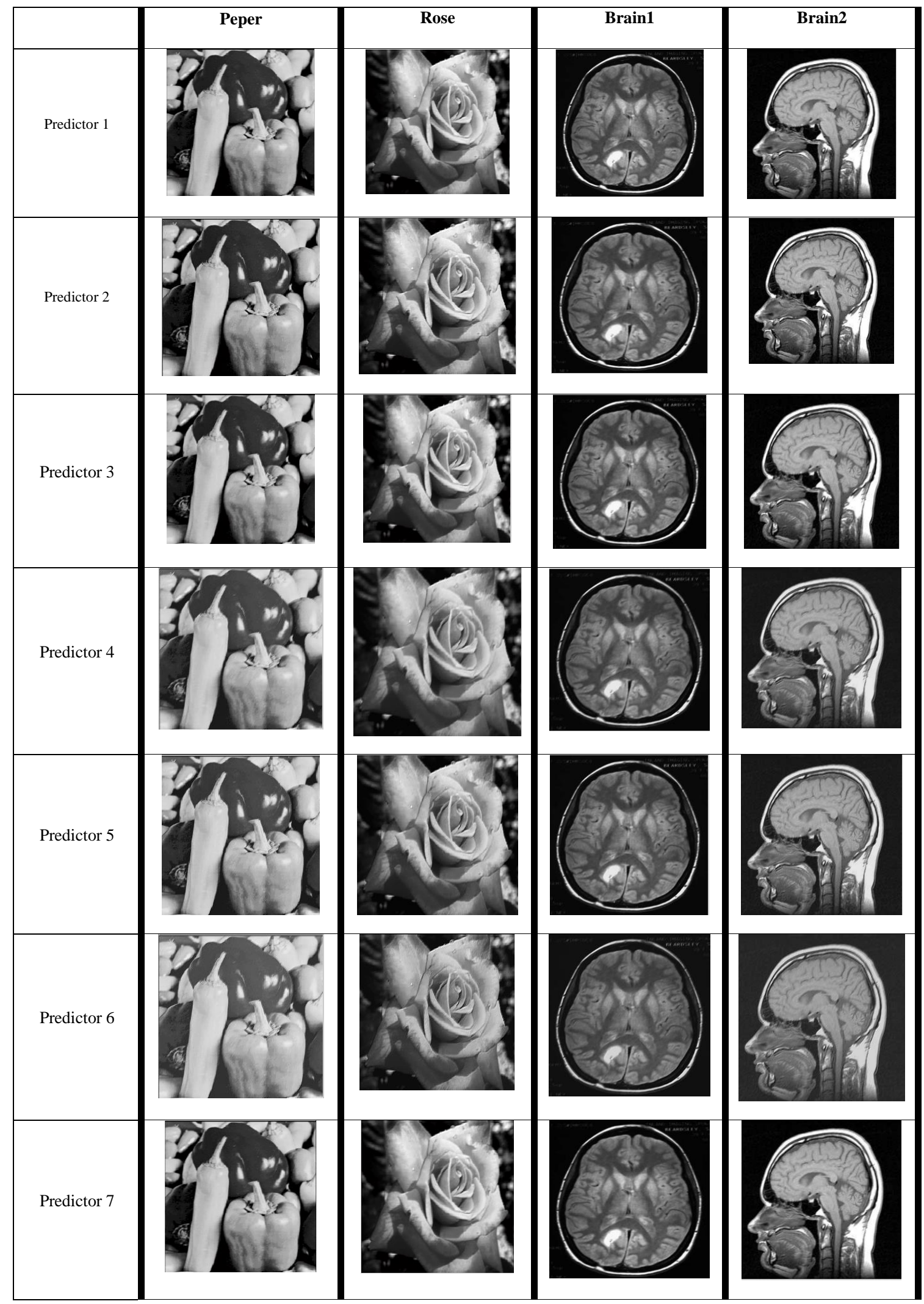

Figure 4 -Resulting Images of the Recursive Prediction using the predictors (1-7) 


\section{Conclusions}

The results obtained from the proposed method showed a promising performance due to the spatial domain utilization to eliminate the redundant image pixels. The best compression results for the tested images was recorded using predictor of order 4; i.e a predictor with four pixels was better than one. Another factor affects the compression ratio in this study is the characteristics of the test image, where high compression rate was recorded for the detailed image (such as rose).

In future, this work can be extended to fit the video compression system

\section{Acknowledgements}

The author would like to thank Mustansiriyah University (www.uomustansiriyah.edu.iq) Baghdad-Iraq for its support in the present work.

\section{References}

[1] Swathy, S., Nahas, J., . "A Study on Medical Image Compression Techniques". International Journal of Innovative Research in Computer and Communication Engineering, vol. 5, no. 4, pp. 8105-8110, April 2017.

Available at: http://www.ijircce.com/upload/2017/april/94_18_A\%20Study.pdf

[2] Al-Timimi, R.T., "Hybrid Lossless Image Compression Using Wavelet Trans-form and Hierarchical non Linear Prediction”. Al-Mustansiriyah Journal of Science, vol. 28, no. 2, pp.150$155,2018$.

Available at: http://mjs.uomustansiriyah.edu.iq/ojs1/index.php/MJS/article/view/510 doi: http://dx.doi.org/10.23851/mjs.v28i2.510

[3] Ghadah , Al-K. ,Haider, Al-M., "Lossless Compression of Medical Images using Multiresolution Polynomial Approximation Model". International Journal of Computer Applications, vol. 76, no. 3, pp. 42-38, 2013.

Avaibleat:https://www.researchgate.net/publication/260845363_Lossless_Compression_of_Medi cal_Images_using_Multiresolution_Polynomial_Approximation_Model doi: $10.5120 / 13230-0659$

[4] Faisel, G.M. Color Image Compression Based on DWT. Ph.D. Thesis. College of science, University of Baghdad ,Iraq, 2006.

[5] George, L. E., Dhannon,B.N., "Image Compression Using Polynomial and Quadtree Coding Techniques". International Journal of Scientific \& Engineering Research, vol. 4, no. 11, pp. 2229-5518, 2013.

Available at:

https://pdfs.semanticscholar.org/407c/8d8d74a4ade58bd5078e9fea03b39ae14be7.pdf

[6] Rasha, Al-T., Image compression using Enhancement Polynomial Prediction Coding. Master thesis, University of Baghdad, Iraq, 2015.

Available at:

https://www.researchgate.net/publication/330092970_Image_Compression_Using_Enhancement_ Polynomial_Prediction_Coding_Supervised_by

DOI: 10.13140/RG.2.2.18550.45125

[7] George, L. E, Sultan, B., "Image Compression Based on Wavelet, Polynomial and Quadtree". Journal of Applied Computer Science \& Mathematics, vol. 11, no. 5, pp. 20-15, 2011.

Available at:

https://www.researchgate.net/publication/267713119 Image Compression Based On Wavelet Polynomial and Quadtree

[8] Al-Timimi, R.T., "Lossy Images Compression Based on Multiresolution". Al-Mustansiriyah Journal of Science, vol. 29, no. 2, pp.126-134, 2018.

Available at: http://mjs.uomustansiriyah.edu.iq/ojs1/index.php/MJS/article/view/242 doi: http://doi.org/10.23851/mjs.v29i2.242

[9] Ghadah , Al-K., George, L. E., "Fast Lossless Compression of Medical Images based on Polynomial”. International Journal of Computer Applications, vol. 70, no. 15, pp. 28-32, 2013. Availableat:https://www.researchgate.net/publication/269666825_Fast_Lossless_Compression_of _Medical_Images_based_on_Polynomial 
DOI: $10.5120 / 12039-7999$

[10] Al-Timimi, R.T., "Lossy Image Compression based on Differential Coding and Linear Polynomial". Journal of College of Education, no.5, pp.433-442, 2016.

Available at: http://edumag.uomustansiriyah.edu.iq/index.php/edumag/article/view/523.

[11]Wang, J., Lossless Medical Image Compression . Ph.D. Thesis. University of Wollongong, 2011. https://pdfs.semanticscholar.org/1e00/1a773b9331f31a17d180eadadc3f9aa4ba82.pdf

[12] Ghadah, Al-K., Intra and inter frame compression for video streaming. $\mathrm{PhD}$ thesis, Extraunion,UK, 2010.

Available at: https://scholar.google.com/citations?user=bPGGWd4AAAAJ\&hl=en

[13]Rasha, Al-T., Ghadah , Al-K., "Image Compression Using Hirarchical Linear Polynomial Coding”, International Journal of Computer Science and Mobile Computing, vol. 4, no. 1, pp. 112-119, 2015.

Availableat:https://www.researchgate.net/publication/328432678_IMAGE_COMPRESSION_US ING_HIRARCHICAL_LINEAR_POLYNOMIAL_CODING

[14] Al-Timimi, R.T., "Bit Plane Slicing, Wavelet and Polynomials Mixing for Image Compression". Iraqi Journal of Science, vol. 60, no. 11, pp.2497-2505, 2019.

Available at: http://scbaghdad.edu.iq/eijs/index.php/eijs/article/view/1148

DOI: 10.24996/ijs.2019.60.11.22 